\title{
On-chip analysis of $C$. elegans muscular forces and locomotion patterns in microstructured environments
}

\author{
Shazlina Johari, ${ }^{a}$ Volker Nock, ${ }^{b}$ Maan M. Alkaisi ${ }^{b}$ and Wenhui Wang*c \\ Received (in $X X X, X X X)$ Xth $X X X X X X X X X 20 X X$, Accepted $X$ th $X X X X X X X X X 20 X X$ \\ ${ }_{5}$ DOI: $10.1039 /$ b000000x
}

The understanding of force interplays between an organism and its environment is imperative in the biological process. Noticeably scarce from the study of $C$. elegans locomotion is the measurement of the nematode locomotion forces together with other important locomotive metrics. To bridge the current gap, we present the investigation of $C$. elegans muscular forces and locomotion metrics (speed, amplitude and

10 wavelength) in one single assay. This assay uses Polydimethylsiloxane (PDMS) micropillars as force sensing elements and, by variation of the pillar arrangement, introduces microstructure. To show the usefulness of the assay, twelve wild-type $C$. elegans sample worms were tested to obtain a total of 4665 data points. The experimental results lead to several key findings. These include: (1) maximum force is exerted when the pillar is in contact with the middle part of the worm body, (2) C. elegans locomotion

15 forces are highly dependent on the structure of the surrounding environment, (3) the worms' undulation frequency and locomotion speed increases steadily from the narrow spacing of 'Honeycomb' design to the wider spacing of 'Lattice' pillar arrangement, and (4) C. elegans maintained their natural sinusoidal movement in the microstructured device, despite the existence of PDMS micropillars. The assay presented here focuses on wild type $C$. elegans, but the method can be easily applied to its mutants and 20 other organisms. In addition, we also show that, by inverting the measurement device, worm locomotion behaviour can be studied in various substrate environments normally unconducive to flexible pillar fabrication. The quantitative measurements demonstrated in this work further improve the understanding of C. elegans mechanosensation and locomotion.

\section{Introduction}

25 The nematode Caenorhabditis elegans (C. elegans) has served predominantly as an excellent model organism for studying mechanosensation and locomotion at the neuronal level. This is mainly due to its simple nervous system with only 302 neurons and a fully-sequenced genome. The core of $C$. elegans 30 mechanosensation and locomotion is centred within the body mechanics of the worm, which are regulated by their body wall muscles. As shown in Fig. 1, there are six touch receptors along the C.elegans body responsible for mechanosensation. These touch receptors are situated next to the body cuticle, which runs 35 along the body wall muscles ${ }^{1}$. C. elegans body wall muscles consist of 95 muscle cells, which are arranged in four quadrants along the length of the worm body. Each muscle typically has three to five muscle arms, which act as pathways for the muscles to receive stimulation from the nerve ${ }^{2}$. The coupling between the 40 muscles and the outer cuticle of the worm body will induce the contraction of the dorsa-ventral part that leads to the generation of sinusoidal waves which propagate along the body length ${ }^{3}$. These dorsal-ventral muscles push the worm against its surrounding thus generating force thrust and enabling 45 locomotion. The movement pattern of C.elegans depends on both the material and geometric properties of the surrounding environment ${ }^{4,5}$. Various substrates have been used to quantify worm locomotion. These include agar plates ${ }^{6}$, gels of varying stiffness $^{7}$, buffer solutions ${ }^{8}$, gelatine ${ }^{8}$, and saturated particles ${ }^{9,} 10$. 50 Locomotion behaviour can also be influenced by natural aging ${ }^{11}$, external exposure to toxins and drugs ${ }^{12-14}$, or through the manipulation of specific genes ${ }^{15-18}$.

Since C. elegans is a genetically amendable organism, mutated worms with different number of muscles arms can be obtained.

${ }_{55}$ According to Wang et al. ${ }^{19}$, a positive relationship exists between the number of muscle arms and the amplitude of the waves that C. elegans exhibit during locomotion. Since the amplitude wave is generated from the contraction force of the dorsal-ventral muscles, it is of significant interest to investigate how these 60 muscles generate force from different numbers of muscle arms. In addition, further insight into how muscle activity can produce a certain movement pattern is required to better understand the influence of the mechanical properties of the worm body and its physical environment. As the young adult worm size is on the 65 order of approximately $1 \mathrm{~mm}$ in length and $80 \mu \mathrm{m}$ in width, a dedicated force sensor is required to quantify worm forces. Various approaches for measuring forces of $C$. elegans have been reported.

For example, Park et al. ${ }^{20}$ have fabricated a silicon 70 piezoresistive cantilever which was utilized as a force- 


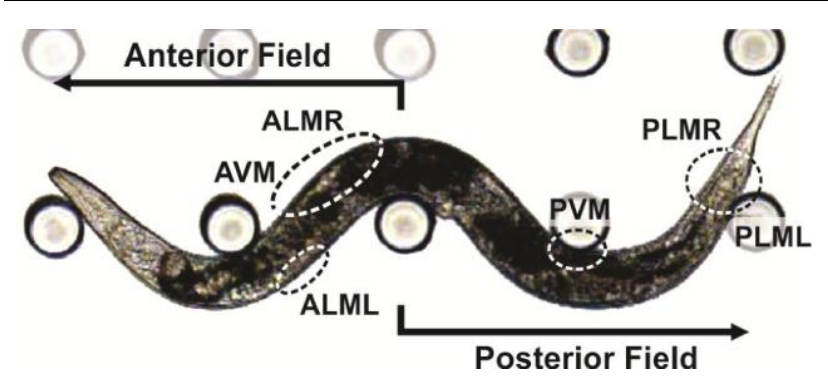

Fig. 1 Micrograph of C. elegans indicating the location of its touch receptor neurons. ALML: Anterior lateral microtubule cell left; ALMR: Anterior lateral microtubule cell right; PLML: Posterior lateral microtubule cell left; PLMR: Posterior lateral microtubule cell right; AVM: Anterior ventral microtubule cell; PVM: Posterior ventral microtubule cell. These neurons are able to detect external forces applied to the body wall muscles and internal forces generated during locomotion. Adapted from Tavernarakis and Driscoll ${ }^{21}$.

displacement measurement system in order to analyse the mechanics of C.elegans body tissue. The indentation method provided by the cantilever limited the worm movement as the worm was partially immobilized on the agar substrate using glue. 5 While this method does capture the peak magnitude of worm forces, it excludes the sensor from measuring continuous force of moving C.elegans. In another work, Doll et al. ${ }^{22}$ have demonstrated the use of microfabricated SU-8 pillars to measure the C. elegans touch sensitivity during locomotion. The device 10 consists of four fixed-guided cantilever arms with gold resistors as strain gauges deployed on the bottom base of the pillar. The applied force from the worm at the pillar tip will cause changes in the strain gauges resistance values. This approach required complex procedures when fabricating the strain gauges. In 15 addition, heat dissipation from the gold resistors might disturb the worm locomotion behaviour ${ }^{23}$. The most recent work was by Liu and co-workers, ${ }^{24}$ where the inclusion of fibre-optics in their microfluidic devices for the measurement of nematode muscular forces has the advantage of providing high sensitivity. This work 20 however requires the integration of fibre optics and was focussed only on $O$. dentatum instead of $C$. elegans.

Recent advances in microfabrication technology and the adoption of microfluidics have made it possible to further investigate C. elegans phenotypic locomotive behaviour ${ }^{25,} 26$. ${ }_{25}$ Several microfluidics-based C. elegans assays have been developed, which include mazes and arrays for learning and mobility behaviour ${ }^{27-29}$, clamps for immobilization and imaging ${ }^{30}$, an olfactory chip $^{31}$, and a droplet-based system for individual movement assays ${ }^{32,} 33$. Microfluidic devices have also been 30 employed as research tools to study C. elegans locomotion patterns and behaviour ${ }^{26,34}$. The latter in particular are relevant to our work on $C$. elegans force measurement as they focused on studying the locomotion behaviour of the nematode using microfabricated Polydimethylsiloxane (PDMS) devices. In 35 particular, a series of sinusoidal microchannels with varying amplitudes and wavelengths was used to match the worm sinusoidal movement ${ }^{35}$. Their suggested methods can be used as a screening system for locomotion phenotypes. As previously mentioned, C. elegans locomotion is highly dependent of its 40 environment. In a different work by Park et al. ${ }^{36}$, it was found that by using microstructured short agar pillars the worm locomotion can be enhanced, particularly when the nematode wavelength matched the micropillars array periodicity.
Here, we expand upon the above concept to investigate ${ }_{45}$ C. elegans locomotion behaviour with regards to forces generated during motion. Previously, we have developed a flexible micropillar-based on-chip system capable of instantly measuring multi-point forces for a worm sample of C. elegans in motion ${ }^{37}$. The current micropillar-based system is able to measure force 50 with a resolution in the order of $\mu \mathrm{Ns}$ for body widths of $80 \mu \mathrm{m}$. The device was microfabricated using PDMS to allow C. elegans to move in a matrix of micropillars inside a channel. By using a vision-based algorithm to detect the pillar deflection, the incident force exerted by a worm can be resolved.

${ }_{55}$ In this paper, we extend our previous work ${ }^{38}$ to the underlying physics of the force pattern generated by C. elegans during locomotion. We demonstrate the correlation between C. elegans locomotion forces and their environment by introducing variation into the microstructured pillar arrangement and spacing. We ${ }_{60}$ further provide evidence that the natural sinusoidal movement of C. elegans remains similar in the device, despite the existence of the PDMS micropillars. Due to the transparency of our device we simultaneously quantify related locomotion parameters, such as the average locomotion velocity, body amplitude and the bending 65 wavelength. Our findings provide further insight into the correlation between locomotion and the generated force patterns, thus demonstrating the device as an enabling technology for research regarding $C$. elegans mechanosensation and locomotion behaviour. Additionally, our device can also be incorporated with 70 PDMS microvalves, which can simplify individual worm selection and manipulation for force measurement ${ }^{39}$. Through automation of valve control, the system has the potential to enable high-throughput nematode force screening in the future.

\section{Experiment}

\section{${ }_{75}$ Device design}

The microfabricated device (Fig. 2) consists of a $9 \times 9 \mathrm{~mm}$ square chamber with a matrix of PDMS pillars and a glass coverslip enclosing the top. Each pillar can be regarded as an individual cantilever beam which functions as a force-measuring unit ${ }_{80}$ dedicated to one individual $C$. elegans. At any given time a nematode will be in contact with 10 to 20 pillars, depending on worm size and pillar spacing. The pillar dimensions were set to be $100 \mu \mathrm{m}$ height and $60 \mu \mathrm{m}$ diameter. The chamber is enclosed by four sidewalls which are $20 \mu \mathrm{m}$ higher than the pillar tips. ${ }_{85}$ This is to ensure that the glass coverslip does not touch the pillar tips when the pillars are deflected. In addition, it protects the worm and pillars from contamination. The front and rear faces of the test section are open and function as the worm loading and extraction zones. Two different pillar configurations were used in 90 this work (Fig. 3). The first arrangement is called the 'Honeycomb' (HC) design, in which the pillars are arranged in a hexagonal formation (Fig. 3a and 3c). The second arrangement is called the 'Lattice' (LC) design, where the pillars are arranged in a square lattice grid structure (Fig. $3 \mathrm{~b}$ and 3d). These 95 configurations were chosen as they mimic the worm's natural environment by providing an array of obstacles used to investigate worm locomotion behaviours ${ }^{29}$. In addition, both configurations have been previously ${ }^{36,40}$ compared regarding their effect on locomotion, with square-post (LC) arrays found to 
(a)

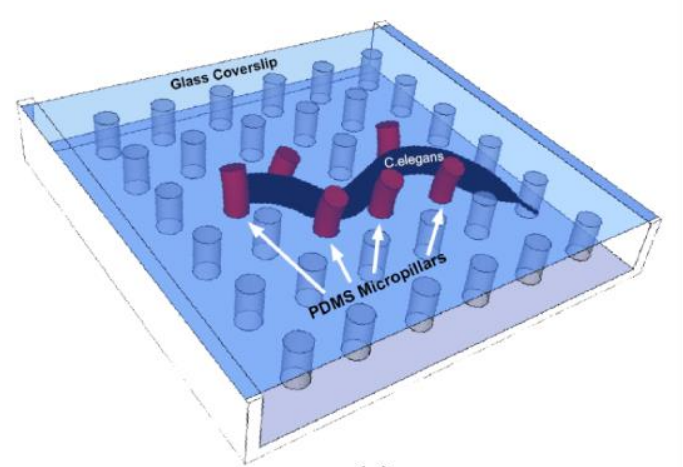

(b)

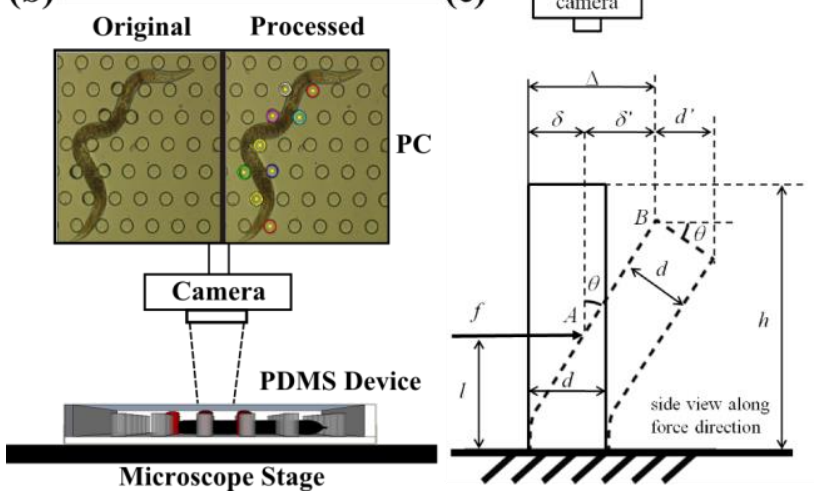

Fig. 2 (a) Sketch of C. elegans movement deflecting the micropillars in the PDMS device. (b) Experimental setup comprising the PDMS device on the microscope stage with a camera connected to a PC for recording of worm videos. (c) Schematic of the deflected pillar for C. elegans force measurement model $^{37}$.

enhance nematode locomotion compared to hexagonal arrays ${ }^{36}$. For both pillar configurations, there are two different layouts for each arrangement. The first uses a pillar centre-to-centre distance of $110 \mu \mathrm{m}$ and the second is arranged with a spacing of $140 \mu \mathrm{m}$.

\section{${ }_{5}$ Device fabrication}

The microfluidic devices used in this work were fabricated using standard soft lithography procedure ${ }^{37}$. Since the pillar height is not equal to the chamber depth, a two-layer photoresist mould was used. SU-8 photoresist was spin-coated onto a silicon wafer 10 to first create a base of $20 \mu \mathrm{m}$ thick SU-8 2025. This was then soft-baked at $65{ }^{\circ} \mathrm{C}$ for 1 minute followed by 3 minutes at $95{ }^{\circ} \mathrm{C}$ and exposed in a Suess MA6 mask aligner to form the chamber outline. The second layer of SU-8 2100 with a thickness of $100 \mu \mathrm{m}$ was coated on top of the first layer after postbake. The 15 wafer was soft-baked again for 5 minutes at $65^{\circ} \mathrm{C}$ followed by 20 minutes at $95^{\circ} \mathrm{C}$. A second mask was used to expose a combination of chamber outline and the micropillar arrays. After development, PDMS pre-polymer was prepared by mixing Sylgard 184 (Dow Corning) base:curing agent in a 10:1 w/w 20 ratio. The pre-polymer was thoroughly mixed and degassed to remove any air bubbles. The surface of the SU-8 mould was treated by exposure to trimethylchlorosilane (TMCS, Sigma Aldrich) vapour for 2 hours to facilitate de-molding. Following this, the polymer was cast over the SU-8 mould and cured on a

25 hot-plate at $80^{\circ} \mathrm{C}$ for 1 hour. Cured devices were carefully peeled off and cross-linked for a further 4 hours at $80{ }^{\circ} \mathrm{C}$. This additional bake was performed to ensure that the pillar structure had fully hardened and that material properties had stabilized. Individual

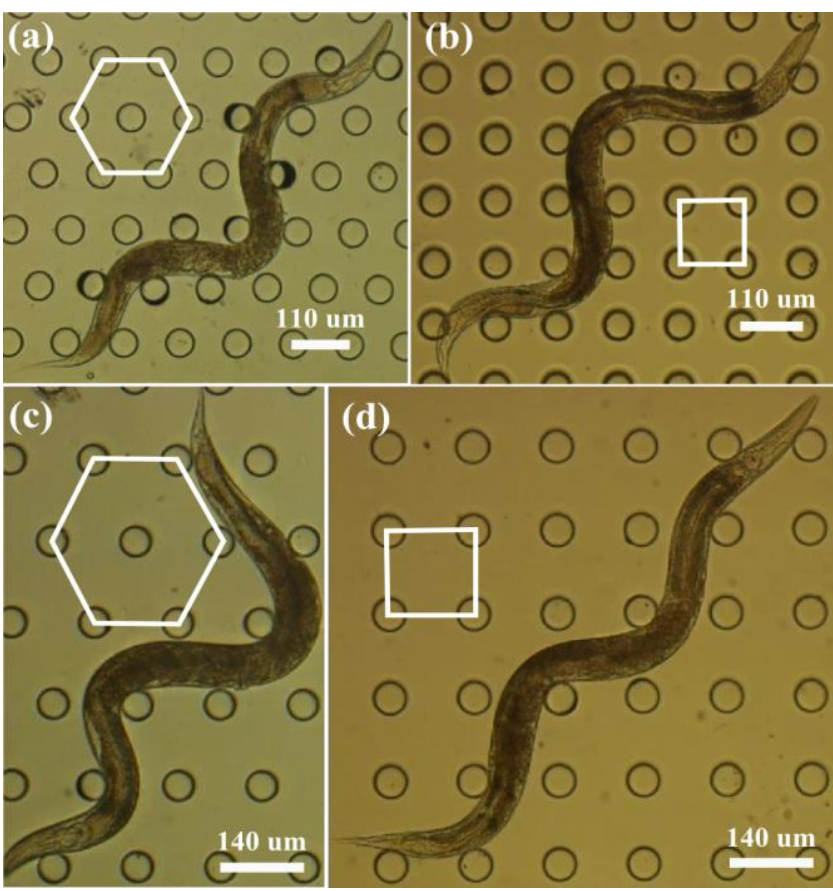

Fig. 3 PDMS device with two sets of micropillar configurations. (a) \& (c) 'Honeycomb' (HC) design with pillar centre-to-centre distances of $110 \mu \mathrm{m}$ and $140 \mu \mathrm{m}$, respectively. (b) \& (d) 'Lattice' (LC) design with pillar centre-to-centre distances of $140 \mu \mathrm{m}$.

devices were then cut out using a scalpel and placed on 30 microscope slides for handling.

\section{Materials and experimental setup}

Wild-type (N2) C. elegans used in this work were cultured and prepared according to Brenner ${ }^{15}$. Prior to nematode loading, the PDMS device surface was treated using a corona wand (Electro${ }_{35}$ Technic Products) to ensure the device is hydrophilic. Then, to mimic the nematode natural habitat, the device was filled with de-ionized (DI) water to provide a moisturized environment for worm locomotion. The amount of water was carefully controlled in order to produce a very thin layer of liquid with a negligible 40 thickness relative to the worms' body width throughout the chamber. The experiments were conducted using young adult C. elegans, where each worm was individually transferred from the Petri dish and very carefully placed in the device loading area. Worms selected for the experiment had similar body width ${ }_{45}$ diameters of approximately $80 \mu \mathrm{m}$. Each worm was given at least 5 minutes to physically adjust to the environmental change.

A schematic of the experimental setup is shown in Fig. 2. Worm movement through the pillar matrix was imaged using a Nikon Eclipse 80i fluorescence microscope under 10x 50 magnifications in bright-field mode. Videos obtained at $5 \mathrm{~Hz}$ were processed offline using a custom image-processing algorithm ${ }^{41,}{ }^{42}$. The device was washed thoroughly with water before each new worm sample in order to avoid crosscontamination.

\section{${ }_{55}$ Results and discussion}

Four different sets of test structures were used in this study: HC design with a pillar centre-to-centre distance of $110 \mu \mathrm{m}, \mathrm{LC}$ pillar 

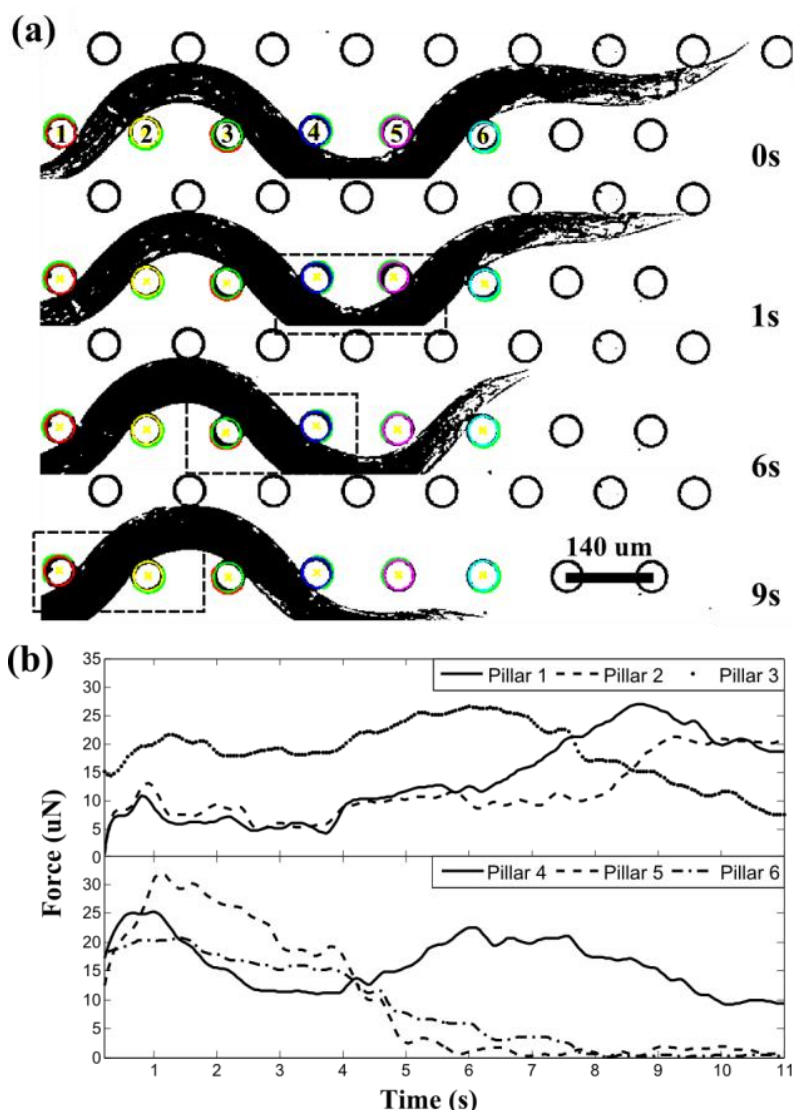

Fig. 4 (a) Image sequence of $C$. elegans motion in $\mathrm{HC}$ design with a pillar centre-to-centre distance of $140 \mu \mathrm{m}$. Elapsed time (sec) is indicated on the lower right of each frame. The worm is in contact with 6 different measurement pillars with the middle part of the body outlined. (b) The associated force magnitude generated on each of the pillars in the anterior (top) and posterior (bottom) field.

structure with a pillar spacing of $110 \mu \mathrm{m}$, HC layout with $140 \mu$ mpillar centre-to-centre distance and LC design with $140 \mu \mathrm{m}$ pillar spacing. Each pillar design was tested using three different nematodes. Through visual observation we found that 5 the worms quickly adapt to the new environment as they continuously generated smooth crawling motion without major difficulty. During the course of motion observed, different body parts of the worm, from head to tail, were in contact with the micropillars. This indicates that different muscles are interacting 10 with the environment.

C. elegans body wall muscles are divided into four quadrants covering the length of the body, i.e. two ventral and two dorsal ones $^{43}$. When worms were navigating in between the smaller spacing of $110 \mu \mathrm{m}$ in either HC or LC design, the worm body 15 contacted the pillars both dorsal and ventral. This is in contrast with the wider spacing of $140 \mu \mathrm{m}$ where the worms normally were in contact with either the dorsal or ventral part of their bodies. Note that in this paper, only the pillars that were observed to experience the most deflection and were in contact with the 20 worm's body throughout the entire recorded video were chosen for force measurement.

\section{Force measurement of individual C. elegans}

We first analysed the force pattern generated by each worm when (a)
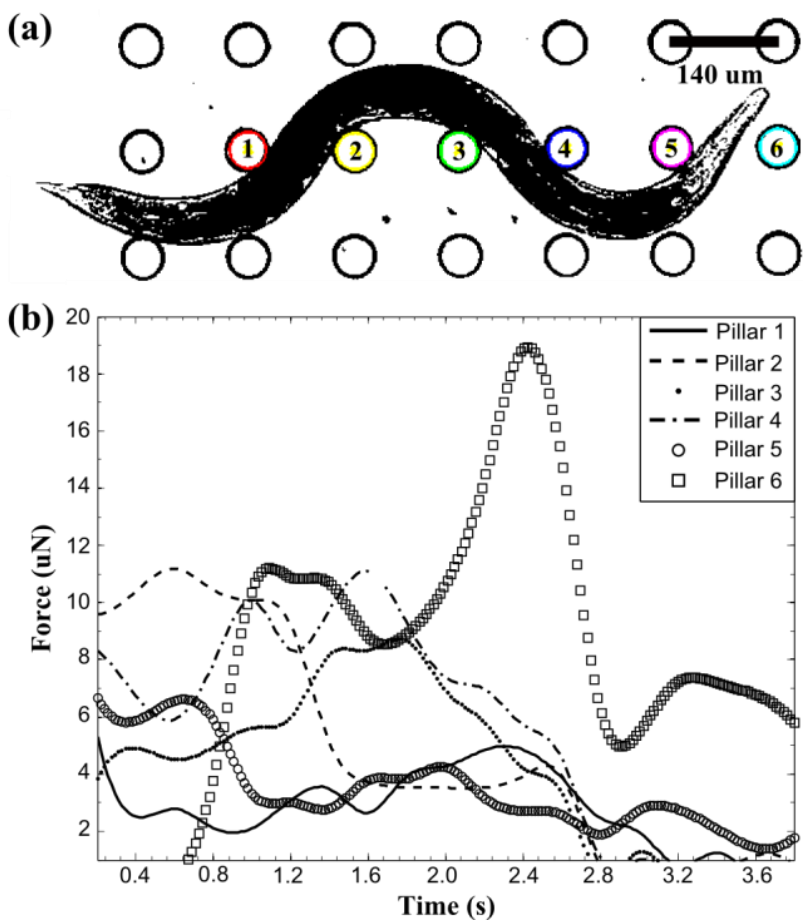

Fig. 5 (a) C. elegans motion in LC design with the pillar centre-to-centre distance of $140 \mu \mathrm{m}$. The worm is in contact with 6 different measurement pillars and (b) force magnitude generated on each of the pillars.

25 moving inside the device. Figure 4 shows the force pattern generated by a single worm using the $\mathrm{HC}$ design structure with a pillar centre-to-centre distance of $140 \mu \mathrm{m}$. The worm was observed to exhibit a constant sinusoidal movement pattern which was in contact with six different pillars (Pillar 1 to Pillar 6) 30 during the movement excerpt of $\sim 7$ seconds shown here.

For the first second, pillars 4 and 5, which were at the middle part of the worm body produced the maximum force, as depicted in the force plot in Fig. 4. This pattern is evidence that throughout the motion, any pillar that is in contact with the worm's mid-body 35 part will register the greatest force. Our findings agree with the theoretical analysis reported by Shen et al. ${ }^{44}$, which stated that $C$. elegans concentrates most of its bending forces around the middle part of its body, especially when crawling. The sinusoidal shape generated by the worm body is split into two parts: the 40 anterior field and the posterior field. From the force plot, it can be seen that the distributed force is not equal or symmetrical along the worm body length. The posterior field was observed to exert greater locomotion force compared to the anterior field. We can verify that highly variable and continuous force levels were 45 produced by the worms in motion, which is in accordance with the biological anatomy of C. elegans.

We then investigated the force pattern generated using the LC design structure with the same pillar centre-to-centre distance of $140 \mu \mathrm{m}$, as depicted in Fig. 5. It was found that the maximum ${ }_{50}$ force was also exerted when the pillar was in contact with the middle part of the worm body. The average generated force is $40 \%$ lower when moving inside the LC structure with a maximum force of $18.86 \mu \mathrm{N}$ compared to the $\mathrm{HC}$ design in which the worm was capable of exerting a maximum force of $31.33 \mu \mathrm{N}$. ${ }_{55} \mathrm{We}$ speculate that this is due to the offset provided from the pillar arrangement in the $\mathrm{HC}$ structure. Hence it might be hypothesized 

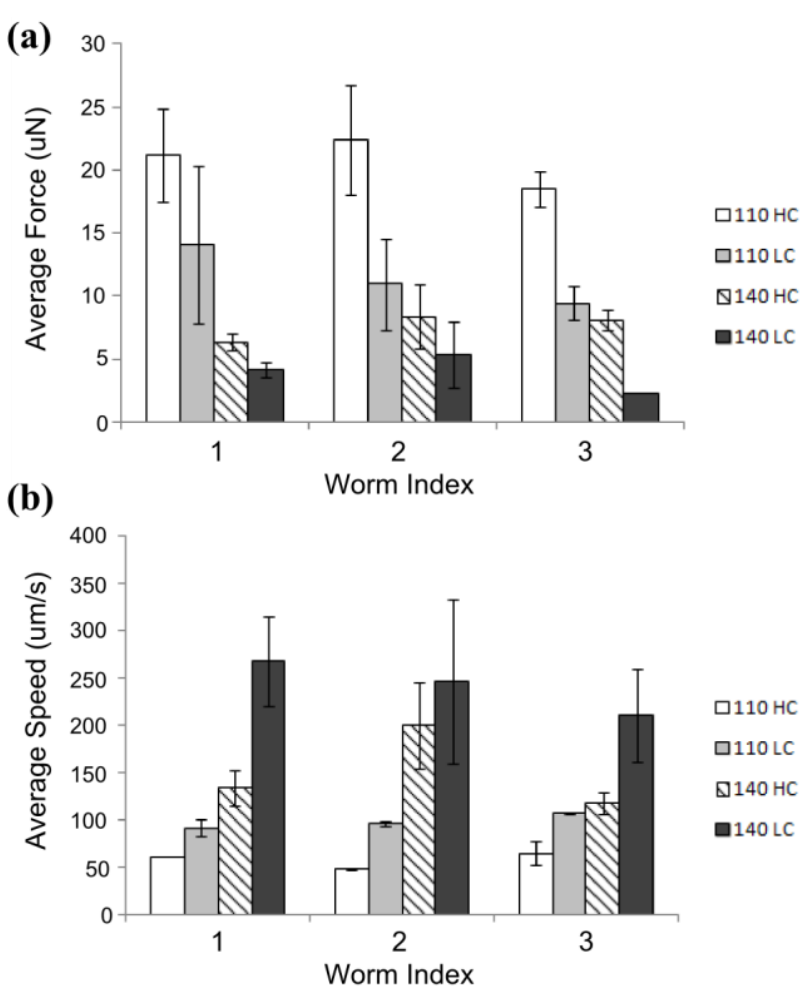

Fig. 6 (a) Average force for twelve different C. elegans measured in the four different device designs. (b) Average worm speed from each device.

that the complex pillar arrangement of the HC design compared to the simple matrix structure from the LC design provided more obstacles to the worm movement and forced the worm to exert larger forces when pushing against the measurement pillars.

5 In addition to the different pillar configuration in the microstructured environment, we also decreased the pillar centreto-centre distance by $30 \mu \mathrm{m}$. The narrow spacing between the pillars often resulted in more worm-pillar contact during locomotion. Figure 6a shows a force plot of a single $C$. elegans in $10 \mathrm{a} \mathrm{HC}$ design structure with the pillar centre-to-centre distance of $110 \mu \mathrm{m}$. During this 8 second period, the worm made contact with ten different measurement pillars which were distributed along the body length. The maximum force was generated from the posterior field of the worm body at pillar 1. In an attempt to 15 correlate the worm body wall muscle with the generated force, we compared the position of the pillar to the touch receptor neurons. From this analysis it appears that the location of pillar 1, which is very close to the PVM touch receptor (Fig. 1), might be the main contributing factor.

20 Although the aim of this work was not focussed on the microstructure of $C$. elegans mechanosensation, our findings can be used as a platform to further examine this in the future. From the reduced pillar spacing, we found that the maximum force exerted during motion increased to two-fold if compared to the 25 larger pillar distance spacing. The narrow spacing between the pillars provided more resistance for the worm to propel itself during movement, hence producing bigger force thrusts. This provides further evidence regarding force thrust and thus supports the result reported by Berri et al. ${ }^{7}$, who found that C. elegans 30 modulates its frequency of undulations as a function of the resistivity of the physical environment.

The same narrow spacing was implemented on the lattice pillar structure. An 8 second video segment was processed for an individual C.elegans, which generated an average force of ${ }_{35} 4.7 \mu \mathrm{N}$ (video and graph available in ES I). During this movement period, the worm started with forward movement and changed its direction by reversing backward at $1.6 \mathrm{~s}$. This motion increased the generated force, especially around pillar 1 and 4 where a maximum force of $13 \mu \mathrm{N}$ was exerted. It might be 40 hypothesized that since this particular part of the worm's body is close to the AVM and PVM touch receptor neurons, the associated body wall muscles around it are highly sensitive to changes around its body especially during the reversing motion. The exerted forces (around pillar 1 and 4) then dropped to $9 \mu \mathrm{N}$ 45 and were observed to be constant throughout the entire remaining motion sequence.

\section{Force comparison for different pillar structures}

During the experiments, 3 worm samples were used for each 50 device, with each worm's locomotion recorded for the duration of 4 to 8 seconds. For the $\mathrm{HC}$ design, at least six pillars were observed to make contact with the worm's body and the number of pillars increases when the pillar centre-to-centre distance decreases. The same applies to the LC pillar design arrangement.

${ }_{55}$ Worms generated higher forces when they were in the $\mathrm{HC}$ structure, and the narrow grid spacing in this particular layout resulted in a $64 \%$ increased average force compared to the bigger pillar spacing of $140 \mu \mathrm{m}$. We also found that when inside the LC pillar arrangement, the worms' locomotion forces were smaller 60 compared to when moving inside the HC structure. Although the main reason behind this is currently still unclear, we believe that the simplicity of the pillar arrangement in the lattice design might be a major contributing factor. In addition, the wider gap between the adjacent pillars $(140 \mu \mathrm{m}$ pillar centre-to-centre distance) ${ }_{65}$ provides less restriction to movement of the worms as they exert very low average forces during motion.

We then examined the measured forces of all the worms for a different set of test structure. Figure 6 a shows the average force collected from 12 different worms (based on 4207 data points in 70 total). It is apparent that there are distinct qualitative differences in the measured locomotion forces. From this we can verify that C. elegans locomotion forces are highly dependent on their environment. The complex arrangement and narrow micropillars spacing pose more resistive obstacles during movement, thus 75 requiring the worm to generate greater force thrusts while navigating in between the pillars. As the spacing increases, we observed that the generated forces decrease. During this period, the number of pillars that are in contact with the worm decreases, and the nematode was observed to readily weave its way through 80 the micropillars. Although forward locomotion is the main movement pattern of the nematode, we found that on a few occasions the worms exhibited other movement behaviours. For instance, worms were observed to reverse their motion especially when changing their movement direction. (See video clip in ${ }_{85}$ Electronic Supplementary Information).

\section{Measurement of other C. elegans locomotion parameters}

Worms were also observed to move at different speeds when inserted in a different pillar layout. To further validate these 
observations, we measured the locomotion speed using the ImageJ software package ${ }^{45}$ for each individual worm in each of the different arrangements. Figure $6 \mathrm{~b}$ plots the average locomotion speed of 12 individual $C$. elegans (error bars are 5 standard deviations for $n=3$ ) as a function of the device design structure and its pillar centre-to-centre distance.

The wide range of locomotion speeds produced by the worms in different test structures is immediately apparent, especially for the case where the pillar centre-to-centre distance was $140 \mu \mathrm{m}$. 10 As expected, the worm moved the slowest $(57.8 \mu \mathrm{m} / \mathrm{s})$ in the design with the narrowest pillar centre-to-centre distance of $110 \mu \mathrm{m}$. The locomotion speed increases as the spacing increases, with the average recorded speed of $150 \mu \mathrm{m} / \mathrm{s}$ for the pillar centreto-centre distance of $140 \mu \mathrm{m}$.

15 In comparison of the pillar layouts, worms were observed to produce higher speeds when moving in the LC structure compared to the $\mathrm{HC}$ test arrangement. We found that the average speed increased by $39 \%$ in the former design compared to the latter. When comparing the measured speed with the worm 20 locomotion on conventional agar plate our findings conclude that the worm movement in the wider spacing of $140 \mu \mathrm{m}$ between the pillar centre-to-centre distances matched the typical movement behaviour observed for natural agar without any microstructured pillars. It thus seems likely that the gap between the pillars, which 25 is equal to the worm's body width $(\sim 80 \mu \mathrm{m})$, provides easier navigation for the worm compared to the narrow spacing. This suggests that when moving inside the LC structure with the wider spacing, worm motion is enhanced with a very low force of less than $8 \mu \mathrm{N}$ relative to the narrow spacing. In contrast, the 30 nematodes have to exert larger forces when navigating through the obstacles provided by the narrow spacing hence slowing down their motion speed.

We also found that the worms' undulation frequency depends on the micropillar arrangement and spacing. Figure 7a shows the 35 undulation frequency ( $f=$ speed/wavelength) measured on agar plates and in the four device structures. It can be seen that, while the frequency decreases for the narrow HC design compared to agar plates, it rises to values larger than those observed on plates for the wider spacing arrangements. This variation in the 40 undulation frequency verifies that the worm responds to different geometric constraints imposed by the pillar arrangement through changes in the period at which it flexes its dorsal-ventral body wall muscles.

Two of the important indicators of worm adaptability to its 45 environment are sinusoidal body amplitude and wavelength during motion. We measured these parameters for every single worm and plotted the average values (based on 36 data points from all test structures) in Fig. 7b. For the narrow pillar spacing the amplitude ranges from 150 to $180 \mu \mathrm{m}$, which is comparable to 50 values observed on agar plates. It increases to 200 to $300 \mu \mathrm{m}$ when worms move inside the pillar designs with wider spacing. We found that in the test configurations all worms show sinusoidal movement similar to their natural environment, while variation of the pillar spacing appears to change their body 55 amplitude. The change of the pillar structure however does not change their body shape as they are able to adapt in both the HC and LC structure similarly. Despite the changes in the pillar arrangement, measurement of the worm body amplitude in these

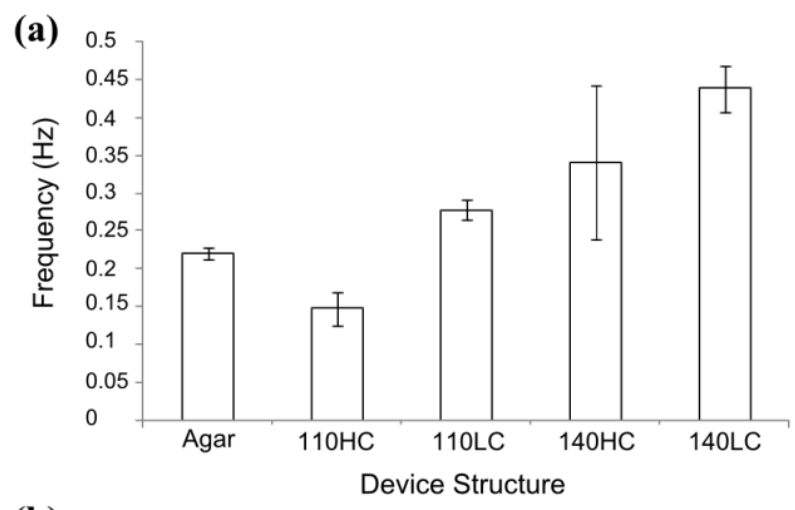

(b)

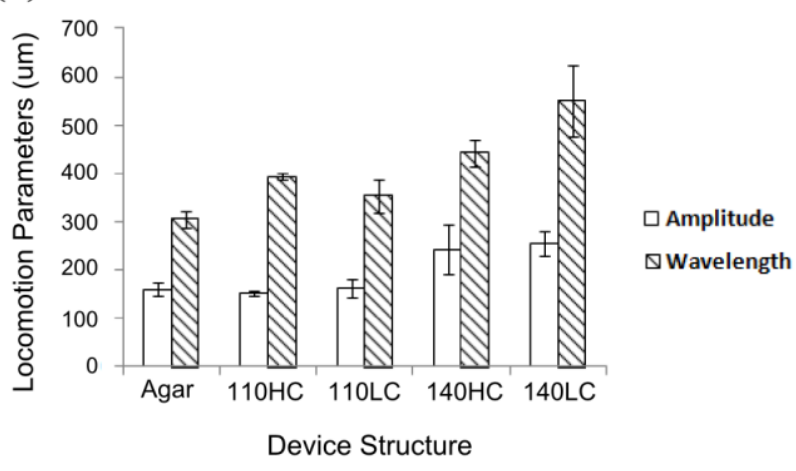

Fig. 7 Comparison of C. elegans undulation frequency on agar plates and in different pillar array devices. Error bars are standard deviations for $n=$ 3. (b) Plot of locomotion amplitude and wavelength for twelve C. elegans samples. Error bars are standard deviations for $n=3$. Values indicate nematode crawling behaviour comparable to data reported for smooth crawling ${ }^{35,44}$.

fits the amplitude ranges reported by Parashar et al. ${ }^{35}$ where 60 smooth movement of C. elegans was reported in the amplitude range of 91 to $225 \mu \mathrm{m}$. This also agrees with the findings by Lockery et. al. ${ }^{40}$ who observed worms crawling easily when their amplitude waveform matched their designated channel amplitude of 100 to $200 \mu \mathrm{m}$.

65 Our results regarding the locomotion parameters and corresponding measured forces agree with the simplified relationship by Lockery et al. ${ }^{40}$. This states that tangential thrust $F$ exerted by a half-wave in undulatory locomotion is proportionally related to the amplitude $A$ and inversely to the 70 wavelength $\lambda$ as given by

$$
F \propto \frac{2 \pi A / \lambda}{\lambda \sqrt{1+(2 \pi A / \lambda)^{2}}}
$$

As shown in Figs. 6 and 7, this model supports the decrease in average force for increasing wavelength $\lambda$ (and almost constant amplitude $A$ ) observed in our experiments for both HC and LC 75 designs at the wider $140 \mu \mathrm{m}$ spacing. Higher forces exerted by locomotion in the narrower devices are due to the increase in pillar-worm contact area and thus friction, which, being a tangential force, retards the motion leading to lower observed speed $^{46}$.

80 Furthermore, the recorded amplitude values are in close agreement with the velocity, in such manner as that worms produce larger amplitudes when they move faster. In regards to 
body wavelength, C. elegans show a similar pattern of positive relationship with the pillar spacing. The wavelengths increase from $350 \mu \mathrm{m}$ when inside the narrow spacing up to $600 \mu \mathrm{m}$ when navigating through the wider spacing. These values suggest that,

5 in the reported microstructured environment, the worms showed similar crawling motion as shown on the agar culture plates.

\section{C. elegans locomotion forces on other substrates}

Since the mechanical and chemical properties of PDMS devices 10 differ from standard plates used for nematode studies we further investigated a modified, substrate-independent version of our measurement technique. As depicted in Fig. 8a, the PDMS sensor device was attached to a glass slide and inverted, so that the pillar tips face towards the substrate surface. A micromanipulator 15 (Sutter Instruments MP-285) was used to control the device movement ensuring that the pillar tips will not collide with the substrate. Though the device configuration is now different, the force measurement model used in this work can still be applied (see Figure S2, ESI).

20 While trying to apply this system to standard agar substrates we encountered two major problems: First, although the positioning of the device slightly above the agar surface was possible, the moisture transferred from the worm's body regularly contaminated the PDMS tips and hence led to reduced image 25 quality. Secondly, liquid film forming between the agar and the tips reduced the total deflection of the pillars due to surface tension effects and thus hindered accurate force measurement.

To our surprise these effects were not observed when the agar plate was substituted for a conventional glass microscope slide 30 covered by a thin layer of liquid. A single worm was again placed on the moisturized glass and the device was controlled by the micromanipulator such that the worm's body touched the pillar tip during movement (Fig. 8b). We found that the worm used the micropillar tips to facilitate its movement and it thus seems likely

35 that the liquid on the glass slide surface enforced swimming motions which made movement less effective ${ }^{44}$. The micropillars on the device assisted the worms by guiding their movement and at the same time deflected to indicate the applied forces. Figure $8 \mathrm{c}$ plots the forces exerted by an example worm over a 40 duration of 7 seconds. The maximum force observed was $9.05 \mu \mathrm{N}$, which is less than a third of the value measured for movement inside the microstructured PDMS device (Fig. 4b). The observed locomotion produced an average force of $3 \mu \mathrm{N}$ (from 350 data points), which was also a factor of two lower 45 compared to movement inside the PDMS device shown in Fig. 5a. This result is consistent with the forces reported by Doll et al. $^{22}$ who measured in a similar manner. The experiment demonstrated above thus shows that our device can be used to measure forces on other substrates, and worm locomotion 50 behaviour in various environments can be investigated further.

While the behaviour of $C$. elegans locomotion has been widely studied, the underlying physics behind the force exerted during its locomotion are still arguably unknown. In their natural habitat, the soil provides a more challenging and complex environment 55 compared to the smooth agar plates typically used in laboratory. The purpose of this study is to provide a better understanding of the worms' natural locomotion forces by reproducing similar/comparable environments. This was achieved by (a)

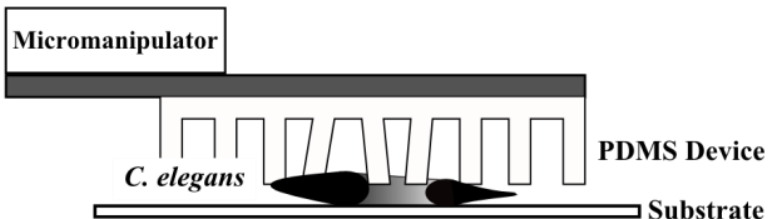

(b)

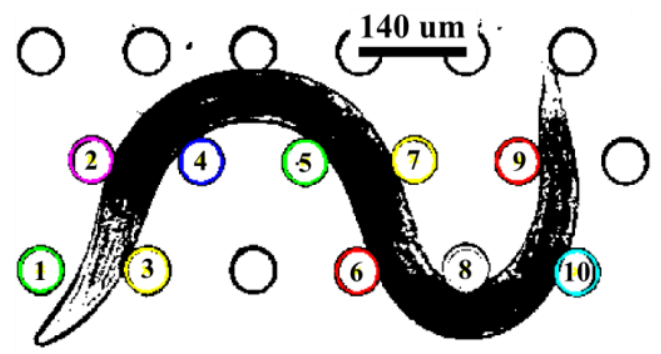

(c)

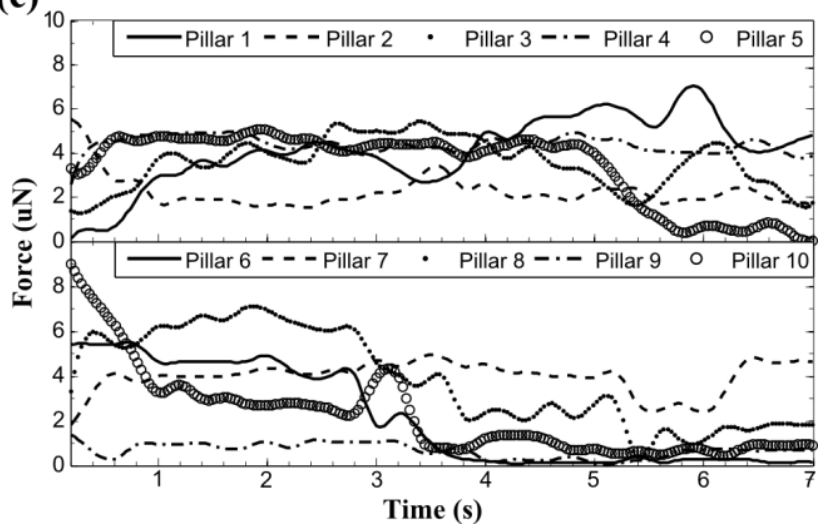

Fig. 8 (a) The PDMS micropillar device is placed on a glass slide which is inverted and affixed to a three-axis micromanipulator. An individual worm is put on the glass slide for force measurement. (b) Pillar layout and associated force pattern produced by the worm on a glass slide. (c) Associated force magnitude measured by each of the pillars in the anterior (top) and posterior (bottom) field.

arranging the PDMS micropillars in different layouts, and 60 varying the spacing between pillars.

The latter was performed mainly to investigate the worm's adaptability and locomotion behaviour in different surroundings. Our main findings suggest that even this simple arrangement of micropillar arrays significantly affects the worm's contraction 65 force, the locomotion speed and the undulation frequency. All these appear to strongly depend on the micropillar spacing and arrangement. One of the most interesting observations is that C. elegans can adapt to the environment by showing similar sinusoidal body shape while navigating in between the 70 micropillars. The worms used the pillars as anchors to push off and increase their locomotion speed, instead of being hindered by the obstacles. These regimes of enhanced locomotion depend on the layout of the micropillars scaled by the length of the nematode. In addition, we also observed changes in frequency, 75 velocity, curvature, and the gait of the worm as a function of the microstructured environment.

The main advantage of our device is its capability to quantify multipoint forces of a moving C. elegans which, to our knowledge has not been reported before. The forces measured 80 during locomotion in the micropillars can also be used to differentiate mutant phenotypes. During movement, $C$. elegans depends on the transduction of their touch receptor neurons in order to navigate its environment. For example, it has been 
reported that mechanosensory mutants (mec-4, mec-10) fail to navigate in short agar pillar structures ${ }^{36}$. Thus, it will be of interest to quantify the force exerted from such mutants and compare it with the forces exhibited by wild type $C$. elegans 5 reported here. The comparison should provide new insight into the connection of the worm's touch receptors with the locomotion system.

Results found here can also be used as a starting point in other C. elegans studies. One example is to investigate the 10 significance/importance of muscle arm number on C. elegans locomotion in microstructured environments. We assume that mutants with different numbers of muscle arms will exhibit similar patterns as reported in this work. However, the quantitative force generated in this case should be 15 increasing/decreasing corresponding on the number of muscle arms. Another example of the potential application of this work is in the investigation of muscle development of worms in different life stages, as each stage displays different body sizes and specific genetic features.

20 Apart from locomotion forces, our device is also capable of conducting concurrent measurement of other locomotion parameters such as speed, amplitude and wavelength. This additional information can be useful to further quantify phenotypic behaviour of $C$. elegans and deepen the understanding 25 of the theory behind worm locomotion forces measured in this work. With such integrated on-chip analysis capabilities, our system offers a promising platform in genetic research and bioengineering applications using small animals models such as C. elegans.

\section{${ }_{30}$ Conclusions}

In this paper we demonstrated simultaneous measurement of C. elegans locomotion forces, amplitude, wavelength and velocity in a single locomotion assay using elastomeric PDMS micropillars as force sensing elements. Two different micropillar 35 layouts were investigated, namely the 'Honeycomb' and 'Lattice' design structure. Our results indicate that the microstructured environment significantly affects the worm's contraction force, locomotion speed and the undulation frequency. All three quantities depend on the micropillar spacing and arrangement. 40 Nematode locomotion forces were greater in the HC structure and locomotion was enhanced inside the LC pillar arrangement. In comparison, the average locomotion forces in narrower spaced pillars increased by 50 to $64 \%$, depending on the layout. We also found that the nematode navigation in the narrow pillar spacing 45 particularly using the $\mathrm{HC}$ design was relatively slow compared to the wider pillar spacing in the LC design. In addition, we have verified that the mid-body of the worm generates the maximum force level, as predicted by theoretical analysis. This on-chip assay can be used as a powerful integrated measurement platform 50 to further investigate the theory behind the worm locomotion forces and C. elegans mechanosensation and its body mechanics.

\section{Acknowledgements}

The authors would like to thank Craig Galilee for providing the C. elegans; and Helen Devereux and Gary Turner for technical 55 assistance. Financial support for S. Johari was provided by
Ministry of Higher Education Malaysia.

\section{Notes and references}

a Department of Mechanical Engineering, University of Canterbury, Christchurch, NEW ZEALAND

${ }_{60} b$ The MacDiarmid Institute for Advanced Materials and Nanotechnology, Christchurch, NEW ZEALAND

c Department of Precision Instruments and Mechanology, Tsinghua

University, Beijing, CHINA. Tel: +86-10-62787338; E-mail: wwh1975@gmail.com

$65 \dagger$ Electronic Supplementary Information (ESI) available: [details of any supplementary information available should be included here]. See DOI: 10.1039/b000000x/

1. M. Chalfie, Biol. Bull., 1997, 192, 125.

70 2. S. J. Dixon and P. J. Roy, Development, 2005, 132, 3079-3092.

3. B. C. Petzold, S.-J. Park, P. Ponce, C. Roozeboom, C. Powell, M. B. Goodman and B. L. Pruitt, Biophys. J., 2011, 100, 1977-1985.

4. N. A. Croll, The behaviour of nematodes: their activity, senses and responses, Edward Arnold, 1970.

75 5. J. T. Pierce-Shimomura, B. L. Chen, J. J. Mun, R. Ho, R. Sarkis and S. L. McIntire, Proc. Natl. Acad. Sci. U.S.A., 2008, 105, 20982-20987.

6. J. Karbowski, C. J. Cronin, A. Seah, J. E. Mendel, D. Cleary and P. W. Sternberg, J. Theo. Biol., 2006, 242, 652-669.

7. S. Berri, J. H. Boyle, M. Tassieri, I. A. Hope and N. Cohen, HFSP J., 2009, 3, 186-193

8. C. Fang-Yen, M. Wyart, J. Xie, R. Kawai, T. Kodger, S. Chen, Q. Wen and A. D. T. Samuel, Proc. Natl. Acad. Sci. U.S.A., 2010, 107, 20323-20328.

9. S. Jung, Phys. Fluid., 2010, 22, 031903

85 10. G. Juarez, K. Lu, J. Sznitman and P. E. Arratia, Europhys. Lett., 2010, 92, 44002 .

11. L. A. Herndon, P. J. Schmeissner, J. M. Dudaronek, P. A. Brown, K. M. Listner, Y. Sakano, M. C. Paupard, D. H. Hall and M. Driscoll, Nature, 2002, 419, 808-814.

90 12. G. L. Anderson, W. A. Boyd and P. L. Williams, Environ. Toxicol. Chem., 2001, 20, 833-838.

13. W. A. Boyd and P. L. Williams, Environ. Toxicol. Chem., 2003, 22, 2768-2774.

14. W. A. Boyd, R. D. Cole, G. L. Anderson and P. L. Williams, Environ. Toxicol. Chem., 2003, 22, 3049-3055.

15. S. Brenner, Genetics, 1974, 77, 71-94

16. L. Brundage, L. Avery, A. Katz, U.-J. Kim, J. E. Mendel, P. W. Sternberg and M. I. Simon, Neuron, 1996, 16, 999-1009.

17. T. A. Starich, R. K. Herman and J. E. Shaw, Genetics, 1993, 133, 527-541.

18. D. M. Miller, C. J. Niemeyer and P. Chitkara, Genetics, 1993, 135, 741-753.

19. W. Wang, Y. Sun, S. J. Dixon, M. Alexander and P. J. Roy, JALA, 2009, 14, 269-276

105 20. S.-J. Park, M. B. Goodman and B. L. Pruitt, Proc. Natl. Acad. Sci. U.S.A., 2007, 104, 17376-17381.

21. N. Tavernarakis and and M. Driscoll, Annu. Rev. Physiol., 1997, 59, 659-689.

22. J. C. Doll, N. Harjee, N. Klejwa, R. Kwon, S. M. Coulthard, B. Petzold, M. B. Goodman and B. L. Pruitt, Lab Chip, 2009, 9, 14491454.

23. S.-J. Lee and C. Kenyon, Curr. Biol., 2009, 19, 715-722. 
24. P. Liu, D. Mao, R. J. Martin and L. Dong, Lab Chip, 2012, 12, 34583466.

25. H. Wen and J. Qin, Sci. China Chem., 2012, 55, 484-493.

26. A. Ben-Yakar, N. Chronis and H. Lu, Curr. Opin. Neurobiol., 2009,

5 19, 561-567.

27. J. Qin and A. R. Wheeler, Lab Chip, 2007, 7, 186-192.

28. H. Ma, L. Jiang, W. Shi, J. Qin and B. Lin, Biomicrofluidics, 2009, 3, 044114-044118.

29. S. Pandey, A. Joseph, R. Lycke and A. Parashar, ABB, 2011, 2, 409-

10415.

30. S. E. Hulme, S. S. Shevkoplyas, J. Apfeld, W. Fontana and G. M. Whitesides, Lab Chip, 2007, 7, 1515-1523.

31. N. Chronis, M. Zimmer and C. I. Bargmann, Nat. Methods, 2007, 4, 727-731.

15 32. W. Shi, H. Wen, Y. Lu, Y. Shi, B. Lin and J. Qin, Lab Chip, 2010, 10, 2855-2863.

33. D. Ying, K. Zhang, N. Li, X. Ai, Q. Liang, Y. Wang and G. Luo, Biochip J., 2012, 6, 197-205.

34. W. Shi, H. Wen, B. Lin and J. Qin, in Microfluidics, ed. B. Lin,

20 Springer Berlin Heidelberg, 2011, vol. 304, ch. 145, pp. 323-338.

35. A. Parashar, R. Lycke, J. A. Carr and S. Pandey, Biomicrofluidics, 2011, 5, 024112-024119.

36. S. Park, H. Hwang, S.-W. Nam, F. Martinez, R. H. Austin and W. S. Ryu, PLoS ONE, 2008, 3, e2550.

25 37. A. Ghanbari, V. Nock, S. Johari, R. J. Blaikie, X. Chen and W. Wang, J. Micromech. Microeng., 2012, 22, 095009.

38. S. Johari, V. Nock, M. M. Alkaisi and W. Wang, Proceedings of the 16th International Conference on Miniaturized Systems for Chemistry and Life Sciences, Okinawa, 2012.

30 39. S. Johari, V. Nock, M. M. Alkaisi and W. Wang, Mater. Sci. Forum, 2012, 700, 182-187.

40. S. R. Lockery, K. J. Lawton, J. C. Doll, S. Faumont, S. M. Coulthard, T. R. Thiele, N. Chronis, K. E. McCormick, M. B. Goodman and B. L. Pruitt, J. Neurophysiol., 2008, 99, 3136-3143.

35 41. A. Ghanbari, V. Nock, R. J. Blaikie, X. Chen, J. G. Chase and W. Wang, Proceedings of the 6th IEEE Conference on Automation Science and Engineering, Toronto, 2010.

42. A. Ghanbari, V. Nock, R. J. Blaikie, J. G. Chase, X. Chen, C. E. Hann and W. Wang, IJCAT, 2010, 39, 137-144.

40 43. G. M. Benian and H. F. Epstein, Circulation Research, 2011, 109, 1082-1095.

44. X. N. Shen, J. Sznitman, P. Krajacic, T. Lamitina and P. E. Arratia, Biophys. J., 2012, 102, 2772-2781.

45. ImageJ Image Processing and Analysis in Java,

45 http://rsb.info.nih.gov/ij/.

46. J. Gray, Q. J. Microsc. Sci., 1953, s3-94, 551-578. 